\title{
Expect the Unexpected: A Case of Isolated Eosinophilic Meningitis in Toxocariasis
}

\author{
Christian Sick Michael G. Hennerici \\ Department of Neurology, University Hospital Mannheim, Mannheim, Germany
}

\section{Key Words}

Eosinophilic meningitis · Toxocariasis · Parasitic zoonosis

\begin{abstract}
We present the case of a young police officer suffering from headache without other neurological symptoms caused by isolated eosinophilic meningitis, which resulted from an infection with Toxocara cati, along with a discussion of the differential diagnosis.
\end{abstract}

(C) 2014 S. Karger AG, Basel

\section{Case Report}

A previously healthy 27-year-old white police officer presented to our emergency department with frontoparietal headache of a dull character lasting for 3 days and maximum intensity in the early hours of the morning. With the exception of a slight sense of feeling sick, which was interpreted by the patient as a flu-like infection, no further clinical symptoms, in particular no signs of meningeal irritation such as photophobia, increased noise sensitivity, nausea, vomiting, nuchal rigidity or fever, were reported. His anamnesis was free regarding journeys abroad, diarrhea or tick bite, but the patient owns 2 cats, of which one has recently undergone an anthelmintic vaccination.

After the initial presumptive diagnosis of sinusitis could be ruled out, no focal deficits were found on neurological examination. Cranial magnetic resonance imaging (MRI) showed no evidence of cerebrovascular, inflammatory or tumorous lesions. X-ray examination of the thorax, ultrasonic testing of the abdomen, ophthalmologic and otolaryngologic examination, and whole-body MRI revealed no pathological findings, in particular no signs of lymphoma or other neoplasias. 
The initial white blood cell count of 5,600/ $\mu$ land the levels of CRP with less than 2.90 $\mathrm{mg} / \mathrm{l}$ were as normal as the erythrocyte sedimentation rate of $4 \mathrm{~mm}$ within $1 \mathrm{~h}$. The only conspicuous finding was a slightly increased relative count of eosinophilic granulocytes in differential blood count of $6.6 \%$ (normal range $<6 \%$ ). Serum electrophoresis of was normal, and we found no increased IgE immunoglobulin fraction. Spinal tap at the day of admission showed a pleocytosis with 700 cells/ $\mu$ l, but with normal protein $(385 \mathrm{mg} / \mathrm{l})$, normal glucose $(51 \mathrm{mg} / \mathrm{dl})$ and normal lactate $(1.91 \mathrm{mmol} / \mathrm{l})$ levels. At this time, there was no sign of disturbed blood-brain barrier or an intrathecal immunoglobulin production. After symptomatic analgetic treatment (flupirtine $1 \times 400 \mathrm{mg}$ /day) with mild improvement of headache but without development of neurological signs, a second lumbar puncture showed protein, glucose and lactate levels still within normal ranges, the cell number declined to $360 / \mu \mathrm{l}$, but now intrathecal IgM production was detected. Manually counted differential blood cells showed an increase of eosinophils to $10 \%$.

As can be seen in figure 1, microscopic cytological examination revealed a pleocytosis (700 cells $/ \mu \mathrm{l}$ ) with predominantly eosinophilic granulocytes $(64 \%)$, but also, in a lesser proportion, lymphocytes $(25 \%)$ and monocytes $(11 \%)$. Therefore, we diagnosed eosinophilic meningitis, but at this point, the etiology was uncertain. No parasitic organism was found in the feces. In cerebrospinal fluid (CSF), polymerase chain reaction for herpes simplex virus 1 and 2 (HSV1 and HSV2), cytomegalovirus, varicella-zoster virus, EpsteinBarr virus, human herpes virus 6, JC virus, enteroviruses and Toxoplasma gondii revealed no pathological findings. Antibody levels [enzyme-linked immunosorbent assay (ELISA)] in CSF and serum were unremarkable for HSV1, HSV2, cytomegalovirus, varicella-zoster virus, Epstein-Barr virus, enteroviruses, measles, mumps, European tick-borne encephalitis, Borrelia burgdorferi, aspergillus, candida, Cryptococcus neoformans, T. gondii, trichinellosis, Echinococcus granulosus, Echinococcus multilocularis, filariasis, strongyloidiasis, cysticercosis, angiostrongyliasis and gnathostomiasis. As can be seen in table 1, for toxocariasis, a borderline antibody titer in the serum (0.43) was found (Swiss Tropical and Public Health Institute Basel, Switzerland), while antibodies in the CSF (normal range: $<0.50$ ) were negative in the first examination.

In the follow-up after 4 weeks, the patient spontaneously recovered from his headache and remained neurologically normal. The suspected diagnosis of eosinophilic meningitis caused by toxocariasis could be confirmed by positive antibody titers of 1.46 in the serum and 0.74 in CSF after a third lumbar puncture. At this point, cell count in the CSF had decreased to $35 / \mu \mathrm{l}$ with only $5 \%$ eosinophilic granulocytes. Therefore, we resigned to initiate a specific therapy with diethylcarbamazine, mebendazole or albendazole, even though we had a clear diagnosis now.

\section{Discussion}

Human toxocariasis is a parasitic zoonosis caused by the larval stages of Toxocara canis and T. cati, the common roundworm of dogs and cats, respectively. Seroprevalence in apparently healthy adults of urban areas of Western countries is reported to be 2-5\% [1], with children more often affected than adults. Humans are infected by the accidental ingestion of Toxocara eggs or encapsulated larvae contained in the raw tissues of paratenic hosts like cows or chicken [2]. Two severe clinical syndromes are classically recognized: caused by larva migration through large organs, visceral larva migrans is a systemic disease, while in ocular larva migrans the disease is limited to the eyes and the optic nerve [3]. Clinical involvement of the central nervous system is thought to be very rare and may lead to 
a variety of neurological disorders such as eosinophilic meningitis [4], eosinophilic meningoencephalitis [5], eosinophilic myelitis [6], cerebral vasculitis [7], optic neuritis [8], epileptic seizures [9] and probably cognitive [10] and behavioral disorders [11]. Depending on the localization of the infection in the central nervous system, affected patients show different symptoms such as severe headache in our patient, fever, oversensitivity to light or noise, nuchal rigidity, weakness, confusion, tiredness and visual impairment, but also focal neurological deficits like ataxia, rigor, weakness of limbs, accentuated deep tendon reflexes or dysesthesia have been described. Manifestations of toxocariasis in the peripheral or autonomous nervous [12] system is rare and only few cases of radiculitis with clinical findings of reduced tendon reflexes, muscle weakness and muscle fasciculation were reported.

Diagnosis of central nervous system involvement of toxocariasis is based on unspecific clinical neurological investigation, peripheral eosinophilia and eosinophilic pleocytosis in the CSF. Confirmation requires detection of specific antibodies or detection of $T$. canis or $T$. cati DNA in the CSF. In the presence of focal symptoms, MRI should be used and in case of cerebral vasculitis cerebral angiography. Finding larvae in the CSF would be considered as a definitive diagnostic tool, but this is considered very rare. In either blood or the CSF, eosinophilia can reach $70 \%$ in toxocariasis. Peripheral blood eosinophilia can be observed without CSF eosinophilia and vice versa as in our patient. In half of the toxocariasis patients, CSF protein is slightly elevated and glucose levels are usually normal. MRI of toxocariasis patients may show subcortical, cortical or white matter lesions that are hyperintense in T2weighted sequences and show homogenous enhancement after the administration of contrast agents [13]. Immunological methods using ELISA are standard to detect antibodies against $T$. cani or $T$. cati in serum or CSF. Due to the fact that negative serum and positive CSF is an exception, the examination of serum samples is worthwhile in toxocariasis of the central nervous system. In our patient, antibodies were seen earlier in the serum than CSF, but there is no general information about the time course of antibodies in serum and CSF. Unlike the findings in our patient, higher antibody titers in CSF than in serum appear to be the norm. Recommended treatment consists in corticosteroids with or without albendazole and cyclophosphamide pulse therapy, if primary angiitis is confirmed. However, symptomatic treatment of severe headache and fatigue syndromes is often sufficient in a visually selflimiting course with full recovery.

Toxocariasis is not the only cause of eosinophilic meningitis or meningoencephalitis. Especially Angiostrongyliasis cantonensis, which is endemic in Asia, has to be considered in patients with eosinophilic meningitis as an alternative possible cause, mainly acquired by the consumption of contaminated water or raw food. But there are other helminthic infections like gnathostomiasis, schistosomiasis, cysticercosis, baylisascariasis, paragonimiasis, trichinellosis, hydatidosis, strongyloidiasis and filariasis that have to be taken into consideration. Even an allergic aspergillus sinusitis [14], Coxsackie viral meningitis, rickettsial disease, Behçet disease and neurosarcoidosis as well as acute lymphoblastic leukemia [15] have been described as rare causes of isolated eosinophilic meningitis. Therefore, even if, as in our case, a patient presents with isolated headache, no systemic signs of infection and no anamnesis of extraordinary travel activity, as physicians, we always have to expect the unexpected. 
Sick and Hennerici: Expect the Unexpected: A Case of Isolated Eosinophilic Meningitis in Toxocariasis

\section{References}

1 Magnaval JF, Glickman LT, Dorchies P, Morassin B: Highlights of human toxocariasis. Korean J Parasitol 2001;39:1-11.

2 Rubinsky-Elefant G, Hirata CE, Yamamoto JH, Ferreira MU: Human toxocariasis: diagnosis, worldwide seroprevalences and clinical expression of the systemic and ocular forms. Ann Trop Med Parasitol 2010;104:3-23.

-3 Pawlowski Z: Toxocariasis in humans: clinical expression and treatment dilemma. J Helminthol 2001;75:299-305.

-4 Goffette S, Jeanjean AP, Duprez TP, Bigaignon G, Sindic CJ: Eosinophilic pleocytosis and myelitis related to Toxocara canis infection. Eur J Neurol 2000;7:703-706.

5 Hayashi E, Akao N, Fujita K: Evidence for the involvement of the optic nerve as a migration route for larvae in ocular toxocariasis of Mongolian gerbils. J Helminthol 2003;77:311-315.

6 Eberhardt 0, Bialek R, Nagele T, Dichgans J: Eosinophilic meningomyelitis in toxocariasis: case report and review of the literature. Clin Neurol Neurosurg 2005;107:432-438.

7 Helbok R, Brenneis C, Engelhardt K, et al: A rare case of Toxocara canis cerebral vasculitis. Eur J Neurol 2007;14:e49.

-8 Komiyama A, Hasegawa 0, Nakamura S, Ohno S, Kondo K: Optic neuritis in cerebral toxocariasis. J Neurol Neurosurg Psychiatry 1995;59:197-198.

-9 Quattrocchi G, Nicoletti A, Marin B, Bruno E, Druet-Cabanac M, Preux PM: Toxocariasis and epilepsy: systematic review and meta-analysis. PLoS Negl Trop Dis 2012;6:e1775.

$\rightarrow 10$ Richartz E, Buchkremer G: Cerebral toxocariasis: a rare cause of cognitive disorders. A contribution to differential dementia diagnosis. Nervenarzt 2002;73:458-462.

$\checkmark 11$ Vidal JE, Sztajnbok J, Seguro AC: Eosinophilic meningoencephalitis due to Toxocara canis: case report and review of the literature. Am J Trop Med Hyg 2003;69:341-343.

-12 Caldera F, Burlone ME, Genchi C, Pirisi M, Bartoli E: Toxocara encephalitis presenting with autonomous nervous system involvement. Infection 2013;41:691-694.

13 Xinou E, Lefkopoulos A, Gelagoti M, et al: CT and MR imaging findings in cerebral toxocaral disease. AJNR Am J Neuroradiol 2003;24:714-718.

14 Chan YC, Ho KH, Chuah YS, Lau CC, Thomas A, Tambyah PA: Eosinophilic meningitis secondary to allergic Aspergillus sinusitis. J Allergy Clin Immunol 2004;114:194-195.

15 Lee D, Lee SH, Yoon SS, Ahn TB: Eosinophilic meningitis without peripheral eosinophilia. Eur Neurol 2012;67:217-219.

Table 1. Laboratory findings in CSF, serum and blood cell count

\begin{tabular}{lcrc}
\hline & Day 1 & Day 7 & Day 28 \\
\hline CSF & & & \\
Cells, $\mathrm{n} / \mu \mathrm{l}$ & 700 & 360 & 35 \\
$\quad$ Eosinophilic granulocytes, \% & 64 & 40 & 5 \\
Protein, mg/l & 385 & 269 & 275 \\
Glucose, mg/dl & 51 & 53 & 54 \\
Lactate, mmol/l & 1.91 & 1.71 & 1.77 \\
Intrathecal Ig synthesis & none & IgM & IgM, IgG, IgE \\
Differential blood cell count & & & \\
Total white blood cells, $\times 10^{9} / 1$ & 5.60 & 5.65 & 5.77 \\
Eosinophilic, \% & 6.6 & 10.0 & 8.0 \\
Basophilic, \% & 0.4 & 0.5 & 0.3 \\
$\quad$ Neutrophilic, \% & 3.2 & 2.8 & 3.2 \\
Toxocariasis antibody titer & & & \\
$\quad$ Serum & - & 0.43 & 1.46 \\
CSF & - & $<0.50$ & 0.74 \\
\hline
\end{tabular}




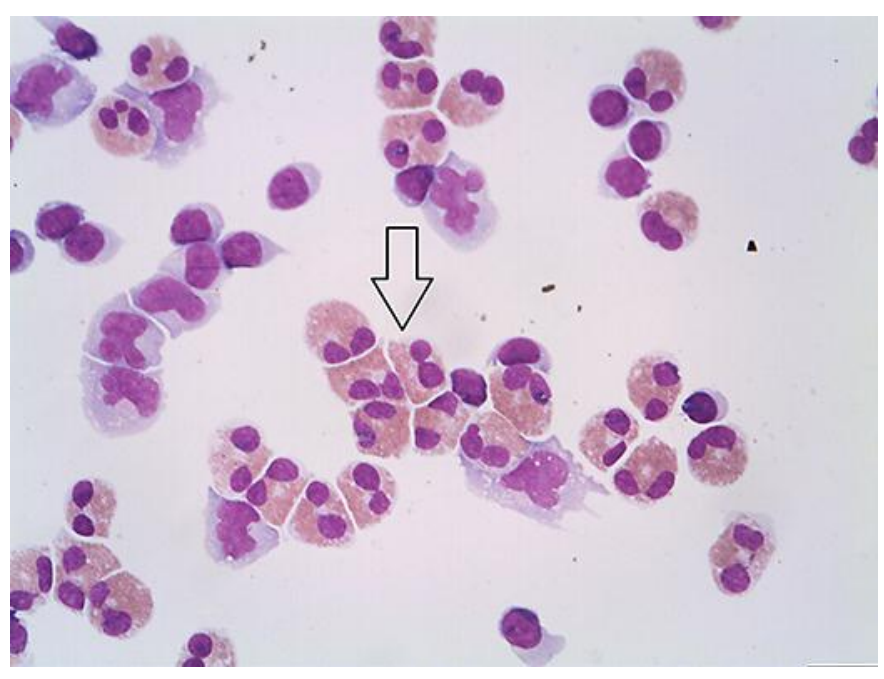

Fig. 1. Microscopic cytological findings of CSF examination with predominantly eosinophilic granulocytes (64\%, marked by an arrow) but also, in a lesser proportion, lymphocytes (25\%) and monocytes (11\%). 